Aim of the study: Pancreatic cancer is one of the most frequent cancers in the world. Only $20 \%$ of patients seem to have disease confined to the pancreas, but in only every second case the tumor turns out to be resectable during surgery. Tumor markers may be a useful tool in differentiating benign from malignant pancreatic tumors and in clinical staging. The purpose of the study is to assess CA 125 utility as a predictor of resectability in pancreatic tumor.

Material and methods: 66 patients were operated on for pancreatic tumor between October 2010 and July 2012. CA 125 concentration was measured in peripheral and portal blood. 57 patients were diagnosed with malignant and 9 with inflammatory tumor. Seven patients had metastases to the liver. Radical surgery was performed in 34 patients.

Results: Significantly higher CA 125 concentration in portal blood was found in the pancreatic cancer than in the inflammatory tumor group (36.5 \pm 99.6 vs. $16.4 \pm 26.5 ; p<0.05$ ). CA 125 concentration in peripheral blood and in portal blood as well of patients with malignant pancreatic tumors and with metastases to the liver was significantly higher than in the group without metastases (146.15 \pm 256.1 vs. $18.5 \pm 17.5 ; p<0.01$ and $147.5 \pm 261.2$ vs. $19.7 \pm 24.3 ; p<0.05$, respectively). CA 125 values in the group without metastases to the liver and in the case of radical surgery were significantly higher in portal than in peripheral blood (19.7 \pm 24.3 vs. $18.5 \pm 17.5 ; p<0.001$ and $13.2 \pm 15.0$ vs. $13.0 \pm 15.2$; $p<$ 0.001 , respectively)

Conclusions: Determination of CA 125 concentration in peripheral blood and in portal blood as well might be a useful tool in differentiating between malignant and inflammatory pancreatic tumors and when decisions on surgery extensiveness are being made.

Key words: CA 125 , portal blood, pancreatic cancer, resectability.

Contemp Oncol (Pozn) 2013; 17 (4): 394-399 DOI: $10.5114 /$ wo.2013.35057

\section{CA 125 concentration in portal blood as a predictor of resectability in pancreatic tumor}

\author{
Krzysztof Szwedziak, Dariusz Szymański, Janusz Strzelczyk
}

Department of General and Transplant Surgery, Medical University of Lodz, Barlicki University Hospital, Lodz, Poland

\section{Introduction}

Pancreatic cancer is one of the most common malignant diseases in the world. It is the fourth leading cause of cancer-related death in men and fifth in women [1]. Pancreatic cancer is characterized by an increasing morbidity rate, which is now around 200000 new cases yearly worldwide [2]. Every year around 3500 people die of it in Poland. Unfortunately, $40 \%$ of patients have distant metastases at the time of diagnosis [3]. A further $40 \%$ of them have locally advanced disease, whereas the remaining $20 \%$ seem to have disease confined to the pancreas $[4,5]$. But in only every second case the tumor turns out to be resectable during surgery, mainly due to vessel invasion or undiagnosed earlier metastases [6]. It makes the prognosis of pancreatic cancer patients bad, with a 5-year survival rate of 15-20\% after curative resection. In case of unresectable tumor the 5-year survival rate is lower and reaches $0.4-4 \%$. Over $90 \%$ of patients after curative resection develop cancer progression within 12-18 months [7, 8]. Despite advances in diagnosis, pancreatic cancer is still diagnosed late. Moreover, staging and prognosis of metastases on the basis of radiological findings tends to be uncertain. The diagnostic accuracy of contrast-enhanced computed tomography (CT) for resectability of pancreatic tumor (defined as tumor-free surgical margins) is about $80 \%[9,10]$. That is why many patients have unnecessary laparotomy, because of tumor nonresectability diagnosed at the time of surgery.

Tumor markers may be useful in differentiating benign from malignant pancreatic tumors and in clinical staging. These are various molecules that can be found in serum, other body fluids or in tissue extracts. Tumor markers make it easier to identify the presence of cancer, to assess patients' prognosis and monitor a patient's response to therapy [11]. Substances used as pancreatic tumor markers may be carbohydrates, mucins, glycoproteins or cytokeratins. One of these markers rarely used for pancreatic cancer is CA 125 (carcinoma antigen 125). It is a member of the mucin family glycoproteins and is produced by tissues originating from epithelium lining fetal cavities. Around $80 \%$ of women with advanced ovarian cancer have elevated levels of CA 125 in their blood serum, making CA 125 a useful tool for detecting ovarian cancer after the onset of symptoms. Its serum half-life is 4 days [12]. CA 125 was detected using the murine monoclonal antibody designated OC125. So far, CA 125 is the only tumor marker that is used for prognosis, disease progression assessment or evaluation of response to chemotherapy in ovarian cancer. CA 125 concentration may also be elevated in other neoplasms including mesotheliomas, endometrial cancer, lung cancer, fallopian tube cancer, breast cancer, pancreatic cancer and gastrointestinal cancers [13, 14]. CA 125 may also be elevated in many benign conditions, such as cirrhosis (64\% of cases), hepatitis (23\%), pancreatitis (25-38\%), jaundice (35\%) and some physiological states (menstruation, first trimester of pregnancy) $[15,16]$. Pancreatic cancer is often accompanied by secondary inflammatory changes caused by pancreatic duct obstruction. 
On the other hand, chronic pancreatitis may lead to cancer development [17]. Co-expression of CA 125 and mesothelin is related to the acquisition of aggressive clinical behavior in pancreatic adenocarcinoma and is associated with worse prognosis. It is interesting that mesothelin is overexpressed in the vast majority of pancreatic adenocarcinomas but not in chronic pancreatitis and normal pancreas $[18,19]$.

The purpose of this study is to assess the utility of CA 125 as a predictor of resectability in pancreatic tumor.

\section{Material and methods}

The study protocol was approved by the local Ethics Committee. 66 patients who underwent elective surgery for tumors of the pancreas and the ampulla of Vater between October 2010 and July 2012 were qualified for the study. None of them had been subjected to either chemo- or radiotherapy. All patients had indications for surgery recognized on the basis of clinical symptoms and imaging techniques including CT or ultrasonography scans of the abdomen. When intraoperatively the tumor was considered as resectable, Whipple procedure, pancreatic distal resection or total pancreatoduodenectomy was performed. The tumors' etiology was determined during a standard histopathology analysis of the surgical specimen. In all cases histopathological analysis confirmed resection RO defined as tumor-free surgical margins. When the pancreatic lesion was found to be nonresectable after laparotomy - because of distant metastases to the liver or local advanced disease - radical surgery was given up. Then biopsy of pancreatic tumor and in some cases biopsy of hepatic tumor were performed. Moreover, in most patients triple bypass (gastrointestinal anastomosis, biliaryenteric anastomosis and enteroenteric anastomosis) or Rouxen-Y gastrointestinal anastomosis was performed.

After laparotomy and identification of the portal triad before manipulation in the vicinity of the tumor - a $4.9 \mathrm{ml}$ (Serum Z/4.9, Sarstedt) blood sample was taken from the portal vein. A similar blood sample was taken simultaneously from the vein in the cubital fossa.

The samples were immediately centrifuged at 3000 rpm and $4^{\circ} \mathrm{C}$ for $10 \mathrm{~min}$. Subsequently, the supernatants were analyzed by the enzyme-linked fluorescence assay (ELFA) and the CA 125 marker concentration was determined (VIDAS CA 125 II). Obtained data were analyzed statistically and descriptively. For measurable values the following statistical analyses were performed: mean $(\bar{x})$ median (Me), standard deviation (Qx), quartile deviation (and variation coefficient ( $\mathrm{\%} \%$ ). In cases where for the analyzed feature only the median and quartile deviation were calculated, the variation coefficient was determined based on these two factors. To determine the dependency between particular features and to compare the frequency of particular varieties of features in analyzed groups the $\chi^{2}$ test was employed. Before the mean values were compared, the agreement of analyzed distributions of measurable features with the normal distribution was checked by means of the $\lambda$-Kolmogorov test. Because the distributions of analyzed features were significantly different from the normal distribution, the Mann-Whitney $U$ test was utilized in order to compare averages in two samples. Because the marker distribution was far from normal, the rank correlation coefficient was applied to determine the dependency between measurable features. Rank correlation coefficient significance was evaluated by Student's $t$-test. Statistically significant were deemed those differences between means (or frequencies) and those dependencies between features for which the calculated test value was equal to or larger than the critical value read out of the appropriate tables, taking into account the number of degrees of freedom and probability $p<0.05$.

\section{Results}

The patients were from 23 to 76 years old (mean $59.3 \pm 10.4$ ) and half of them were over 60.5. There were more men than women (37 vs. 29). The most frequent location of the tumor was the pancreatic head (65\%), followed by the ampulla of Vater (18\%), pancreatic body (11\%) and pancreatic tail (5\%).

The most frequent histopathological diagnosis was pancreatic cancer (46 cases), followed by carcinoma of the ampulla of Vater (11) and pancreatitis (9). In 38 cases of pancreatic cancer the tumor was localized in the head of the pancreas, in 5 cases in the body and 2 tumors were identified in the tail. There was one patient with an entire pancreas subjected to cancerous changes. In the group of malignant tumors were adenocarcinoma ( 52 patients), followed by neuroendocrine tumor (4) and extrapulmonary solitary fibrous tumor (1). Metastases to the liver were present among 5 patients suffering from pancreatic cancer and 2 from carcinoma of the ampulla of Vater. There was no statistically significant relation between the localization of cancerous changes and metastasis to the liver $(p>0.05)$. Frequency of malignant changes was identical among men and women (0.86) and again, this was not statistically significant ( $p>0.05)$.

Analysis of the age of patients suffering from malignant tumors revealed that they were aged from 38 to 76 (mean $61.2 \pm 8.5$ ). Furthermore, those with inflammatory tumor were aged 23 to 67 (mean $47.2 \pm 13.8$ ). The patients suffering from cancer proved to be older than those with pancreatitis and this was significant $(p<0.01)$. The most common surgery performed on the analyzed population was Whipple's procedure (27 patients), followed by Roux-en-Y gastrointestinal anastomosis (13 patients), triple bypasses (7) and pancreatic biopsy or distal pancreatic resection (6 each). There were two cases of Roux-en-Y biliary-enteric anastomosis. Furthermore, there were single patients that underwent peritoneal biopsy, hepatic biopsy, total pancreatoduodenectomy, external drainage of pancreatic cyst and Roux-en-Y anastomosis between the pancreatic cyst and jejunum.

There was no statistically significant dependency between the age of patients and the CA 125 marker concentration in the peripheral as well as portal blood $(p>0.05)$ (Table 1).

The CA 125 marker concentration in the peripheral blood was from $4 \mathrm{U} / \mathrm{ml}$ to $720.5 \mathrm{U} / \mathrm{ml}$ with the average value of $32.24 \pm 89.5 \mathrm{U} / \mathrm{ml}$. Half of the analyzed population displayed concentrations exceeding $13.23 \mathrm{U} / \mathrm{ml}$ and $1 / 4$ of the patients showed values larger than $29.93 \mathrm{U} / \mathrm{ml}$. Respective values for the portal blood were as follows: range from $4 \mathrm{U} / \mathrm{ml}$ to 733.5 $\mathrm{U} / \mathrm{ml}$, average $33.9 \pm 93.6 \mathrm{U} / \mathrm{ml}$ and half of the analyzed population exhibited values not larger than $13.0 \mathrm{U} / \mathrm{ml}$. 
Table 1. Dependency between age and CA 125

\begin{tabular}{lccccc} 
Dependency & \multicolumn{3}{c}{ Peripheral blood } & \multicolumn{3}{c}{ Portal blood } \\
\cline { 2 - 6 } $\begin{array}{l}\text { between } \\
\text { age }\end{array}$ & $\begin{array}{c}\text { Rank correlation } \\
\text { coefficient }\end{array}$ & $\begin{array}{c}t \text {-test } \\
\text { value }\end{array}$ & $p$ & Rank correlation & $t$-test \\
coefficient 125 & -0.004 & 0.030 & $>0.05$ & -0.023 & 0.172
\end{tabular}

Table 2. CA 125 in the peripheral blood with respect to tumor character

\begin{tabular}{|c|c|c|c|c|c|c|c|}
\hline \multirow[t]{2}{*}{ Patients } & \multicolumn{6}{|c|}{ Calculated CA 125 parameters - peripheral blood } & \multirow[b]{2}{*}{$\mathrm{v}(\%)$} \\
\hline & $\min$ & $\max$ & $\bar{x}$ & $\mathrm{Me}$ & SD & Qx & \\
\hline Total & 4 & 720.5 & 32.24 & 13.23 & 89.5 & 10.9 & 277.6 \\
\hline With malignancy & 4 & 720.5 & 34.80 & 14.0 & 95.9 & 10.9 & 275.7 \\
\hline With inflammation & 4 & 74.0 & 16.36 & 8.24 & 22.3 & 3.91 & 136.1 \\
\hline Comparison & \multicolumn{6}{|c|}{$p>0.05$} & \\
\hline
\end{tabular}

Table 3. CA 125 in portal blood with respect to tumor character

\begin{tabular}{|c|c|c|c|c|c|c|c|}
\hline \multirow[t]{2}{*}{ Patients } & \multicolumn{6}{|c|}{ Calculated CA 125 parameters - portal blood } & \multirow[b]{2}{*}{$v(\%)$} \\
\hline & $\min$ & $\max$ & $\bar{x}$ & $\mathrm{Me}$ & SD & Qx & \\
\hline Total & 4 & 733.5 & 33.9 & 13.0 & 93.6 & 9.48 & 275.9 \\
\hline With malignancy & 4 & 733.5 & 36.5 & 14.1 & 99.6 & 9.61 & 273.1 \\
\hline With inflammation & 4 & 81.17 & 16.4 & 5.75 & 26.5 & 4.45 & 161.1 \\
\hline Comparison & \multicolumn{6}{|c|}{$p<0.05$} & \\
\hline
\end{tabular}

Table 4. Comparison of CA 125 in the peripheral blood and portal blood in the whole group of patients and according to the character of the illness

\begin{tabular}{|c|c|c|c|c|c|c|}
\hline \multirow[t]{3}{*}{ Group } & \multicolumn{4}{|c|}{ CA 125} & \multicolumn{2}{|c|}{ Comparison } \\
\hline & \multicolumn{2}{|c|}{ Peripheral blood } & \multicolumn{2}{|c|}{ Portal blood } & \multirow[t]{2}{*}{ z-test value } & \multirow[t]{2}{*}{$p$} \\
\hline & $\bar{x}$ & SD & $\bar{x}$ & SD & & \\
\hline Total & 32.3 & 89.5 & 33.9 & 93.6 & 3.190 & $<0.01$ \\
\hline With malignancy & 34.8 & 95.9 & 36.5 & 99.6 & 3.069 & $<0.01$ \\
\hline With pancreatitis & 16.4 & 22.3 & 16.4 & 26.5 & 0.734 & $>0.05$ \\
\hline
\end{tabular}

Table 5. CA 125 in the peripheral blood of patients with/without metastasis to the liver

\begin{tabular}{|c|c|c|c|c|c|c|c|}
\hline \multirow[t]{2}{*}{ Patients } & \multicolumn{7}{|c|}{ Calculated parameters CA 125 - peripheral blood } \\
\hline & $\min$ & $\max$ & $\bar{x}$ & $\mathrm{Me}$ & SD & Qx & $v(\%)$ \\
\hline Total & 4 & 720.5 & 32.24 & 13.23 & 89.5 & 10.9 & 277.6 \\
\hline With metastasis & 9.99 & 720.5 & 146.15 & 66.06 & 256.1 & 44.9 & 175.2 \\
\hline Without metastasis & 4 & 87.69 & 18.50 & 12.18 & 17.5 & 10.1 & 94.5 \\
\hline Comparison & \multicolumn{7}{|c|}{$p<0.01$} \\
\hline
\end{tabular}

A considerably higher CA 125 concentration was observed in the peripheral blood of patients with malignancies rather than inflammation $(34.8 \pm 95.9 \mathrm{U} / \mathrm{ml}$ vs. $16.36 \pm 22.3 \mathrm{U} / \mathrm{ml})$. However, this difference turned out to be statistically insignificant ( $p>0.05$ ) (Table 2).

Comparison between the two groups of patients (malignancies vs. inflammation) in terms of the CA 125 concentration in the portal blood, in turn, showed a statistically significant difference $(p<0.05)$. A statistically significant higher con- centration in the group of patients with malignancies rather than inflammation was observed $(36.5 \pm 99.6 \mathrm{U} / \mathrm{ml}$ vs. $16.4 \pm 26.5 \mathrm{U} / \mathrm{ml}$, respectively) (Table 3 ).

The CA 125 concentrations in the peripheral and portal blood were compared and the calculated difference was statistically significant in the whole group $(p<0.01)$ and the group with malignancies $(p<0.01)$. Also, we discovered that the CA 125 concentration was significantly higher in the portal than peripheral blood. Such a difference was not 
Table 6. CA 125 in the portal blood of patients with/without metastasis to the liver

\begin{tabular}{|c|c|c|c|c|c|c|c|}
\hline \multirow[t]{2}{*}{ Patients } & \multicolumn{7}{|c|}{ Calculated parameters CA 125 - portal blood } \\
\hline & $\min$ & $\max$ & $\bar{x}$ & $\mathrm{Me}$ & SD & Qx & $\mathrm{v}(\%)$ \\
\hline Total & 4 & 733.5 & 33.9 & 13.0 & 93.6 & 9.48 & 275.9 \\
\hline With metastasis & 8.17 & 733.5 & 147.5 & 57.2 & 261.2 & 48.5 & 177.1 \\
\hline Without metastasis & 4 & 150.1 & 19.7 & 11.6 & 24.3 & 7.5 & 123.1 \\
\hline Comparison & \multicolumn{7}{|c|}{$p<0.05$} \\
\hline
\end{tabular}

Table 7. Comparison of CA 125 in the peripheral blood and portal blood in the whole group of patients and depending on the presence of metastasis to the liver

\begin{tabular}{|c|c|c|c|c|c|c|}
\hline \multirow[t]{3}{*}{ Group } & \multicolumn{4}{|c|}{ CA 125} & \multicolumn{2}{|c|}{ Comparison } \\
\hline & \multicolumn{2}{|c|}{ Peripheral blood } & \multicolumn{2}{|c|}{ Portal blood } & \multirow[t]{2}{*}{ z-test value } & \multirow[t]{2}{*}{$p$} \\
\hline & $\bar{x}$ & SD & $\bar{x}$ & SD & & \\
\hline Total & 32.24 & 89.5 & 33.9 & 93.6 & 3.069 & $<0.01$ \\
\hline With metastasis & 146.15 & 256.1 & 147.5 & 261.2 & 0.016 & $>0.05$ \\
\hline Without metastasis & 18.50 & 17.5 & 19.7 & 24.3 & 3.606 & $<0.001$ \\
\hline
\end{tabular}

Table 8. CA 125 in the peripheral blood of patients depending on the surgery they underwent

\begin{tabular}{|c|c|c|c|c|c|c|c|}
\hline \multirow[t]{2}{*}{ Patients } & \multicolumn{7}{|c|}{ Calculated parameters CA 125 - peripheral blood } \\
\hline & $\min$ & $\max$ & $\bar{x}$ & $\mathrm{Me}$ & SD & Qx & $v(\%)$ \\
\hline Total & 4 & 720.5 & 32.25 & 13.2 & 89.5 & 10.9 & 277.6 \\
\hline Non-radical surgery & 4 & 720.5 & 53.19 & 26.7 & 126.4 & 14.6 & 237.6 \\
\hline Radical surgery & 4 & 87.69 & 13.00 & 9.40 & 15.2 & 3.7 & 115.8 \\
\hline Comparison & \multicolumn{7}{|c|}{$p<0.001$} \\
\hline
\end{tabular}

Table 9. CA 125 in the portal blood of patients depending on the surgery they underwent

\begin{tabular}{|c|c|c|c|c|c|c|c|}
\hline \multirow[t]{2}{*}{ Patients } & \multicolumn{7}{|c|}{ Calculated parameters CA 125 - portal blood } \\
\hline & $\min$ & $\max$ & $\bar{x}$ & $\mathrm{Me}$ & SD & Qx & $v(\%)$ \\
\hline Total & 4 & 733.5 & 33.93 & 13.0 & 93.6 & 9.48 & 275.9 \\
\hline Non-radical surgery & 4 & 733.5 & 58.24 & 21.5 & 134.2 & 13.1 & 230.4 \\
\hline Radical surgery & 4 & 69.68 & 13.20 & 9.3 & 15.0 & 4.01 & 114.0 \\
\hline Comparison & & & & & & & \\
\hline
\end{tabular}

observed in the case of the group of patients with inflammatory changes $(p>0.05)$ (Table 4).

A significantly higher CA 125 concentration was observed in the peripheral blood of patients with metastases to the liver than without them $(146.15 \pm 256.1 \mathrm{U} / \mathrm{ml}$ vs. $18.50 \pm 17.5 \mathrm{U} / \mathrm{ml})$ and this difference was statistically significant $(p<0.01)$ (Table 5).

Comparison between the groups of patients with and without metastases to the liver in terms of the CA 125 concentration in portal blood showed a statistically significant difference as well ( $p<0.05)$. A significantly higher CA 125 concentration was observed among patients with metastases to the liver than without them, with respective averages: 147.5 $\pm 261.2 \mathrm{U} / \mathrm{ml}$ vs. $19.7 \pm 24.3 \mathrm{U} / \mathrm{ml}$ ) (Table 6).

Statistically significant differences in the CA 125 concentration in the peripheral and portal blood were noted in both the whole group $(p<0.01)$ and the group without metastases $(p<0.001)$. Significantly higher values of CA 125 were observed in the portal blood of both aforementioned groups, with averages of $33.9 \mathrm{U} / \mathrm{ml} \mathrm{vs} .32 .24 \mathrm{U} / \mathrm{ml}$, and the group without metastases to the liver: $19.7 \mathrm{U} / \mathrm{ml}$ vs. $18.5 \mathrm{U} / \mathrm{ml}$. In the group of patients with metastases the difference between average CA 125 values in the peripheral and portal blood was not statistically significant (Table 7).

The CA 125 concentrations in the peripheral blood of patients who underwent radical and non-radical surgery were compared and the calculated difference was statistically significant $(p<0.001)$. It turned out that the CA 125 concentration in the peripheral blood of the non-radical surgery group is significantly higher than in the radical surgery group (53.19 $\pm 126.4 \mathrm{U} / \mathrm{ml}$ vs. $13.19 \pm 15.2 \mathrm{U} / \mathrm{ml}$ ) (Table 8). 
Table 10. Comparison of CA 125 in the peripheral blood and portal blood in the whole group and depending on the surgery

\begin{tabular}{|c|c|c|c|c|c|c|}
\hline \multirow[t]{3}{*}{ Group } & \multicolumn{4}{|c|}{ CA 125} & \multicolumn{2}{|c|}{ Comparison } \\
\hline & \multicolumn{2}{|c|}{ peripheral blood } & \multicolumn{2}{|c|}{ portal blood } & \multirow[t]{2}{*}{ z-test value } & \multirow[t]{2}{*}{$p$} \\
\hline & $\bar{x}$ & SD & $x$ & SD & & \\
\hline Total & 32.2 & 89.5 & 33.9 & 93.6 & 3.190 & $<0.01$ \\
\hline Non-radical surgery & 53.2 & 126.4 & 58.2 & 134.2 & 1.514 & $>0.05$ \\
\hline Radical surgery & 13.0 & 15.2 & 13.2 & 15.0 & 3.293 & $<0.001$ \\
\hline
\end{tabular}

Also, the comparison of the CA 125 concentration in the portal blood of patients who underwent either surgery showed a statistically significant difference $(p<0.001)$. Moreover, the CA 125 concentration in the peripheral blood of the patients after non-radical surgery was significantly higher than in the case of a radical operation $(58.24 \pm 134.2 \mathrm{U} / \mathrm{ml}$ vs. $13.2 \pm 15.0 \mathrm{U} / \mathrm{ml}$ ) (Table 9).

A comparison of the CA 125 concentration in the peripheral and portal blood exhibited a statistically significant difference in the whole group and the group of patients after radical treatment $(p<0.001)$. A higher concentration was observed in the portal blood. Such a difference was not observed among patients who underwent non-radical surgery (Table 10).

\section{Discussion}

In this work we describe the prospect of the use of the CA 125 marker to assess pancreatic tumor resectability. Despite advances in radiological imaging techniques, the problem of estimation of pancreatic tumors' radical surgical treatment possibilities still remains enigmatic.

The purpose of the study was to determine whether the measurement of the CA 125 marker not only in the peripheral blood but also in the portal blood might be useful for these purposes. To achieve this, its concentration was determined in the group of patients that underwent surgery because of a pancreatic tumor or ampulla of Vater tumor. Blood samples from the peripheral vein before the surgery and from the portal vein during the operation were collected.

66 patients who were successively admitted to the Clinic because of a pancreatic tumor or ampulla of Vater tumor were subjected to our analysis; thus this choice might be deemed random. Men were in the majority in both the group suffering from cancer (32/57) and the group with an inflammatory tumor (5/9). This results from more frequent occurrence of pancreatic cancer as well as pancreatitis among men [20]. The patients with the malignant tumor were older than those suffering from inflammatory lesions - their mean age was $61.2 \pm 8.5$ and $47.2 \pm 13.8$, respectively - and this difference turned out to be statistically significant. The incidence of pancreatic cancer increases with age and reaches a maximum in the $7^{\text {th }}$ decade, which corresponds to the average age of the population suffering from a malignant tumor (61.2). The worldwide occurrence of pancreatic cancer is 5/100 000/year among women and 8.5/100 000 among men [21].

In the available literature there have not been any reports of CA 125 concentration estimation in the portal blood in the case of pancreatic cancer. However, there are many papers on marker concentration measurements in the peripheral blood. Only a few of them concern the CA 125 marker. Its role is highlighted primarily in the case of ovarian cancer. In our analysis we revealed a significantly higher CA 125 concentration in the peripheral blood of patients with metastases to the liver than of those without metastases $(p<0.01)$. Also, analysis of the CA 125 concentration in the portal blood showed its significantly higher concentration among patients with metastases to the liver $(p<0.05)$. Moreover, among patients without metastases the CA 125 concentration was significantly higher in the portal blood. This might result from its dilution in the peripheral blood and the lack of metastases, which could contribute to an increase in the CA 125 concentration in the peripheral blood. There have been reports on the correlation between the CA 125 expression in pancreatic cancer tissues and worse survival rate $(p<0.001)$, independently of other prognostic factors [22]. Moreover, it is known that the CA 125 expression is stronger in metastasis foci than in the primary tumor $[23,24]$. Data obtained from the CA 125 analysis in terms of liver metastases presence corresponds to the data on the kind of surgery. In case of both non-radical operations and patients without metastases to the liver, the CA 125 concentration was significantly higher in the portal than the peripheral blood. The only limitation imposed on the research might be the lack of study of the dependency of the CA 125 concentration and details of the histopathology analysis. We merely assessed the connection with the histopathological character of the tumors, which crucially influences the patient's prognosis. The aim of this study was mostly to determine the suitability of the marker in intraoperative assessment of tumor resectability in conjunction with objective assessment of clinical staging relying on a search for macroscopically visible changes of metastatic character (mostly to the liver) and assessment of the presence of portal vein invasion, celiac trunk invasion or hepatic hilum invasion. The liver is the most common location of pancreatic cancer metastases and their presence considerably contributes to the high mortality rate [25]. Macroscopically invisible small metastases to the liver are very often present already at the moment of pancreas resection [26]. Also, small pancreatic tumors, of diameter below $2 \mathrm{~cm}$, might be related to the metastases, which can suggest that the propensity to metastases develops very quickly during cancerogenesis and progression of the disease.

In conclusion, determination of the CA 125 concentration not only in the peripheral blood but also in the portal blood might be a useful diagnostic tool to differentiate between malignant and inflammatory pancreatic tumors, since a sig- 
nificantly higher CA 125 concentration in the portal blood of patients with malignant than inflammatory tumors was observed. Moreover, we observed its significantly higher concentration in the portal than peripheral blood in the group of patients with cancerous changes. Significantly higher CA 125 concentration in the peripheral blood of patients with metastases to the liver and significantly higher concentrations in the portal blood in case of the lack of this kind of alterations and when a radical operation was performed might be useful when decisions on surgery extensiveness are being made.

The authors declare no conflict of interest.

\section{References}

1. Krejs GJ. Pancreatic cancer: epidemiology and risk factors. Dig Dis 2010; 28: 355-8.

2. Celiński K, Mądro A. Postępy w diagnostyce i leczeniu raka trzustki. Gastroenterol Pol 2005; 12: 341-6.

3. Jemal A, Siegel R, Ward E, Hao Y, Xu J, Thun MJ. Cancer statistics. CA Cancer J Clin 2009; 59: 225-49.

4. Haller DG. New perspectives in the management of pancreas cancer. Semin Oncol 2003; 30: 3-10.

5. Steinberg WM, Barkin J, Bradley EL 3rd, DiMagno E, Layer P. Workup of a patient with a mass in the head of the pancreas. Pancreas 1998; 17: 24-30.

6. Ballehaninna UK, Chamberlain RS. The clinical utility of serum CA 19-9 in the diagnosis, prognosis and management of pancreatic adenocarcinoma: An evidence based appraisal. J Gastrointest Oncol 2012; 3: 105-19.

7. Maisonneuve P, Lowenfels AB. Epidemiology of pancreatic cancer: an update. Dig Dis 2010; 28: 645-56.

8. Ellison LF, Wilkins K. An update on cancer survival. Health Rep 2010 21: 55-60.

9. Delbeke D, Pinson CW. Pancreatic tumors: role of imaging in the diagnosis, staging, and treatment. J Hepatobiliary Pancreat Surg 2004; 11: 4-10.

10. Magee CJ, Ghaneh P, Neoptolemos JP. Surgical and medical therapy for pancreatic carcinoma. Best Pract Res Clin Gastroenterol 2002; 16: 435-55.

11. Fleisher M, Dnistrian AM, Sturgeon CM, Lamerz R, Witliff JL. Tumor markers: Physiology, Pathobiology, Technology and Clinical Applications. AACC press, Chicago 2002

12. O'Brien TJ, Tanimoto H, Konishi I, Gee M. More than years of CA 125 : what is known about the antigen, its structure and its function. Int J Biol Markers 1998; 13: 188-95.

13. Bast RC, Xu FJ, Yu YH, Barnhill S, Zhang Z, Mills GB. CA 125: the past and the future. Int J Biol Markers 1998; 13: 179-87.

14. Creaney J, Robinson BW. Serum and pleural fluid biomarkers for mesothelioma. Curr Opin Pulm Med. 2009; 4: 366-370

15. Grover S, Koh H, Weideman P, Quinn MA. The effect of the menstrual cycle on serum CA 125 levels: a population study. Am J Obstet Gynecol 1992; 167: 1379-81.

16. Funakoshi Y, Suzuki T. Glycobiology in the cytosol: the bitter side of a sweet world. Biochim Biophys Acta 2009; 1790: 81-94.

17. Mackie CR, Cooper MJ, Lewis MH, Moossa AR. Nonoperative differentiation between pancreatic cancer and chronic pancreatitis. Ann Surg 1979; 189: 480-7.

18. Einama T, Kamachi H, Nishihara H, et al. Co-expression of mesothelin and CA125 correlates with unfavorable patient outcome in pancreatic ductal adenocarcinoma. Pancreas 2011; 40: 1276-82.

19. Argani P, lacobuzio-Donahue C, Ryu B, et al. Mesothelin is overexpressed in the vast majority of ductal adenocarcinomas of the pancreas: identification of a new pancreatic cancer marker by serial analysis of gene expression (SAGE). Clin Cancer Res 2001; 7: 3862-8.
20. Yeo TP, Lowenfels AB. Demographics and epidemiology of pancreatic cancer. Cancer J 2012; 18: 477-84.

21. Michaud DS. Epidemiology of pancreatic cancer. Minerva Chir 2004; 59: 99-111.

22. Streppel MM, Vincent A, Mukherjee R, et al. Mucin 16 (cancer antigen 125) expression in human tissues and cell lines and correlation with clinical outcome in adenocarcinomas of the pancreas, esophagus, stomach, and colon. Hum Pathol 2012; 43: 1755-63.

23. Haridas D, Chakraborty S, Ponnusamy MP, et al. Pathobiological implications of MUC16 expression in pancreatic cancer. PLoS One 2011; 6: 26839.

24. Chen SH, Dallas MR, Balzer EM, Konstantopoulos K. Mucin 16 is a functional selectin ligand on pancreatic cancer cells. FASEB J 2012; 26: 1349-59.

25. Disibio G, French SW. Metastatic patterns of cancers: results from a large autopsy study. Arch Pathol Lab Med 2008; 132: 931-9.

26. Amikura K, Kobari M, Matsuno S. The time of occurrence of liver metastasis in carcinoma of the pancreas. Int J Pancreatol 1995; 17: $139-46$.

\section{Address for correspondence}

Krzysztof Szwedziak MD, PhD

Department of General and Transplant Surgery

Medical University of Lodz, Barlicki University Hospital

Kopcińskiego 22

90-153 tódź, Poland

e-mail: kszwedziak@gmail.com

Submitted: 29.01 .2013

Accepted: $\quad 5.03 .2013$ 